\title{
Characteristics and outcomes of pediatric patients presenting at Cambodian referral hospitals without appointments: an observational study
}

\author{
Mackensie A. Yore ${ }^{1 *}$ D, Matthew C. Strehlow², Lily D. Yan³ , Elizabeth A. Pirrotta ${ }^{2}$, Joan L. Woods ${ }^{4}$, Koy Somontha ${ }^{5}$,
} Yim Sovannra ${ }^{6}$, Lauren Auerbach ${ }^{7}$, Rebecca Backer ${ }^{8}$, Christophe Grundmann ${ }^{5}$ and Swaminatha V. Mahadevan ${ }^{2}$

\begin{abstract}
Background: Emergency medicine is a young specialty in many low- and middle-income countries (LMICs). Although many patients seeking emergency or acute care are children, little information is available about the needs and current treatment of this group in LMICs. In this observational study, we sought to describe characteristics, chief complaints, management, and outcomes of children presenting for unscheduled visits to two Cambodian public hospitals.

Methods: Children enrolled in the study presented without appointment for treatment at one of two Cambodian public referral hospitals during a 4-week period in 2012. Researchers used standardized questionnaires and hospital records to collect demographic and clinical data. Patients were followed up at $48 \mathrm{~h}$ and 14 days after initial presentation. Multivariate logistic regression identified factors associated with hospital admission.

Results: This study included 867 unscheduled visits. Mean patient age was 5.7 years (standard deviation 4.8 years). Of the 35 different presenting complaints, fever (63\%), respiratory problems (25\%), and skin complaints (24\%) were most common. The majority of patients were admitted (51\%), while $1 \%$ were transferred to another facility. Seven patients (1\%) died within 14 days. Follow-up rates were $83 \%$ at $48 \mathrm{~h}$ and $75 \%$ at 14 days. Predictors of admission included transfer or referral from another health provider, seeking prior care for the presenting problem, low socioeconomic status, onset of symptoms within $24 \mathrm{~h}$ of seeking care, abnormal vital signs or temperature, and chief complaint of abdominal pain or fever.

Conclusions: While the admission rate in this study was high, mortality was low. More effective identification and management of children who can be treated and released may free up scarce inpatient resources for children who warrant admission.
\end{abstract}

Keywords: Chief complaints, Developing countries, Emergency medicine, Health systems, Pediatrics

\section{Background}

In low- and middle-income countries (LMICs), an estimated $45 \%$ of all deaths and $36 \%$ of disability-adjusted life years (DALYs) are due to diseases and injuries typically addressed by emergency medical services that are currently lacking in these regions [1]. Among these countries is Cambodia, a lower middle-income nation in Southeast Asia which still

\footnotetext{
* Correspondence: mackensie.yore@gmail.com

'Department of Emergency Medicine, UCSF Fresno Center for Medical

Education and Research, 155 N Fresno St, Fresno, CA 93701, USA

Full list of author information is available at the end of the article
}

suffers residual effects of the Khmer Rouge regime of the 1970s, during which violence, famine, and preventable disease killed approximately two million people and completely dismantled the healthcare infrastructure $[2,3]$.

Developing emergency medicine in LMICs requires a great deal of specialized training for providers. Characterizing the potential emergency department population in LMICs is an important step in creating relevant training for future emergency clinicians. Understanding the distribution of chief complaints focuses provider education on symptoms with the highest burden 
of morbidity and mortality, thereby helping emergency care providers more effectively evaluate and manage patients [4]. Despite the importance of this information, the 2013 Academic Emergency Medicine Consensus Conference found insufficient data on chief complaints for most LMICs [4]. Helping address this knowledge gap, our group previously documented the epidemiology and outcomes of adult patients presenting for unscheduled visits to public hospitals in Cambodia [5]; no studies, however, have thus examined an analogous pediatric population in this setting.

Pediatric patients account for $20-35 \%$ of all emergency department (ED) visits globally [6-8]. Moreover, children present with a unique distribution of chief complaints compared to adults and require different clinical management [7, 9]. To address the specific needs of children presenting to EDs in LMICs, the World Health Organization (WHO) developed guidelines for the care of children with traumatic injuries and acute illnesses in resource-limited settings; however, limited training for diagnosing and treating urgent medical conditions poses challenges to guideline adherence, which may contribute to preventable morbidity and mortality in LMICs [10-15]. The recently announced United Nations Sustainable Development Goals includes a call to reduce preventable mortality among children; strengthening pediatric emergency care capacity in LMICs could help achieve this goal. The present study was conducted to identify the characteristics, chief complaints, management, and outcomes of children presenting for unscheduled visits to two public referral hospitals in Cambodia in order to focus training to improve future care.

\section{Methods}

\section{Study design and setting}

We performed a 4-week prospective, cross-sectional study of unscheduled visits to two government provincial referral hospitals in Cambodia: Sampov Meas Provincial Hospital (SMPH) and Battambang Provincial Hospital (BPH). SMPH and BPH are "CPA Level 3," indicating that obstetric, emergency, and surgical services should be available [16]. SMPH has 162 inpatient beds and recorded 9722 visits in 2012, leading to 6564 admissions. BPH has 220 inpatient beds and recorded 40,825 visits and 14,108 admissions in 2012 [17].

The level of emergency care offered at these two hospitals was similar to that at other Cambodian provincial hospitals at the time of the study. Neither hospital had an active triage system, and BPH had no ED, with patients presenting to a variety of departments for treatment. At $\mathrm{BPH}$, most pediatric patients seeking urgent care came directly to the pediatric ward. At SMPH, the hospital's combined ED/ICU managed patients in serious condition, while patients with milder presentations presented elsewhere. At both facilities, study enrollment was hospital wide.

\section{Selection of participants}

Pediatric patients $(<18$ years $)$ presenting without prior appointments during the 4 weeks in July and August 2012 were invited to enroll; patients presenting for routine check-ups and vaccinations without appointment were excluded. Repeat unscheduled visits were considered separate visits. Most enrollment occurred weekdays, 08:00 to 17:00, times of day with highest patient volume. Patients presented infrequently during evening and weekend hours when hospital staffing was limited. Afterhours visits were included if the patient remained at the hospital the following morning.

\section{Methods and measurements}

A team at each site gathered real-time clinical and demographic data using Research Electronic Data Capture (REDCap) forms [18]. Demographic information and up to three chief complaints were obtained from patients, guardians, and staff. A list of all chief complaints is included in the supplemental material (Additional file 1: Figure S1). Hospital records provided vital signs at presentation, diagnostic tests, treatment interventions, disposition, and discharge dates. Two members of the research team reviewed records for completeness and inconsistencies, which were addressed through repeat interviews and hospital record reviews. Follow-up interviews with patients or their guardians were conducted in Khmer (the local language) at $48 \mathrm{~h}$ and 14 days following the initial visit. Follow-up interviews were in-person if the patient remained hospitalized, or by telephone if discharged, and assessed patient location, survival, and functional status. Patients were considered lost to follow-up after three failed contact attempts on successive days.

\section{Outcome measures}

Primary outcomes included admission, functional impairment, and mortality. Patients are typically treated for their entire hospital stay in the department where they initially presented, regardless of appropriateness; therefore, included interventions were limited to those completed within the first $48 \mathrm{~h}$ of initial presentation, thus identifying services most representative of a standard ED setting. Patients staying overnight were considered admitted. Functional impairment was defined as continued hospitalization, significant pain or limitation in performing daily activities, bed confinement, or coma. 


\section{Data analysis}

Because a complete set of vital signs was not consistently documented at participating hospitals, records with data missing for respiratory rate (RR), blood pressure (BP), or heart rate (HR) were included in multivariate analyses with absent values assumed normal. Records with missing values for all other independent variables were excluded from modeling. Sensitivity analysis showed no significant differences in predictors of admission with the conversion of missing vital signs to normal in multivariate analysis.

Comparisons between outcomes for continuous variables were conducted using Wilcoxon two sample $T$ test, while the chi-square test was used for categorical variables. A multivariate logistic regression was built for the primary outcome of admission using predictors identified through univariate analysis, controlling for age and gender; stepwise methods were not used. Statistical analysis was completed using SAS Enterprise Guide for Windows, version 4.3 (SAS Institute Inc. Cary, NC).

\section{Ethical considerations}

We obtained verbal informed consent from patient guardians or patients themselves if unaccompanied and at least 16 years old. In-person, native-speaking translators obtained consent and conducted interviews in Khmer.

The Institutional Review Boards at Stanford University School of Medicine (IRB-24735) and the Cambodian Ministry of Health approved this study.

\section{Availability of data and materials}

The dataset supporting the conclusions of this article is available in the Dryad repository, https://doi.org/10.5061/ dryad.7v8c4.

\section{Results}

\section{Demographic characteristics}

This 4-week study documented 867 unscheduled pediatric patient visits. Mean patient age was 5.7 years with approximately half $(54.3 \%)$ being male (Table 1$)$. Private vehicles were the predominant mode of transport to the hospitals, with nearly $90 \%$ of patients arriving via motorbike, taxi, or tuk-tuk (motorized three-wheeled rickshaw for hire); arrival by ambulance was infrequent $(2.9 \%)$. More than $69.9 \%$ of patients presented to the hospital with sudden $(<24 \mathrm{~h})$ or recent $(1-3$ days $)$ symptoms (Table 1). About one quarter of patients were either transferred directly from another healthcare facility, typically a health center, or referred by an outside medical provider (e.g., a practitioner private clinic) (Table 1).
Patient presentations and management

For the 867 pediatric patients enrolled, 1615 total chief complaints were recorded, 35 of them distinct. Fever was the most common chief complaint (62.5\% of patients; $33.6 \%$ of complaints), followed by respiratory problems, skin-related complaints, vomiting, and abdominal pain (Fig. 1). Injury represented $2.8 \%$ of complaints (Fig. 1). Chief complaints of abdominal pain and fever resulted in a significantly higher admission rate $(p \leq 0.05)$, while respiratory problems and skin complaints correlated with lower admission rates $(p \leq 0.01)$. Of the seven deaths, chief complaints included respiratory problems (3 children), vomiting (3), fever (2), unconsciousness (1), convulsions/seizures (1), genital bleeding (1), and other (1).

Approximately one quarter of all patient records lacked vital sign data (Table 1). Patients treated and released were less likely to have vital signs recorded than those admitted ( $p \leq 0.05$ ). Abnormal vital signs were more frequent in admitted patients than those treated and released $(p \leq 0.05)$; similarly, admitted patients were more likely to present with pain $(p \leq 0.05)$.

One third of patients received any diagnostic testing, with laboratory tests being the most common (Table 2). Imaging, including chest X-ray, and other diagnostics were performed on less than $3 \%$ of patients (Table 2). The most common intervention within the first $48 \mathrm{~h}$ was medication therapy, administered to $81.6 \%$ of patients (Table 2). Intravenous fluids were provided to $40.7 \%$ of patients. All other interventions were infrequent, each received by less than $5 \%$ of patients (Table 2 ).

\section{Patient disposition and outcomes}

The rate of admission was $51.3 \%$, with a 3 -day median length of stay (Table 3). Less than $1 \%$ of patients were transferred to an outside facility or left the hospital prior to being seen or against medical advice (Table 3). Twenty-two patients (3.1\%) were referred to surgery, $81.8 \%$ of whom were admitted.

Follow-up rates were 82.9 and $74.9 \%$ at $48 \mathrm{~h}$ and 14 days, respectively (Table 3 ). Seven deaths were documented, for a $1.1 \%$ overall 14 -day mortality (Table 3 ). Two of the seven deaths occurred within $24 \mathrm{~h}$ of presentation; the rest occurred after admission, one of whom died after discharge. No deaths were documented in patients who were treated and released.

Morbidity was measured by asking patients or guardians whether the patient had returned to baseline functioning. By $40 \mathrm{~h}$ and 14 days post-visit, 49.7 and $93.2 \%$ of patients, respectively, had returned to baseline functioning (Table 3).

\section{Admission multivariate logistic regression model}

A multivariate logistic regression model showed increased admission risk was associated with referral from another health provider $(\mathrm{OR}=4.3$, CI 2.4-7.8), direct transfer from 
Table 1 Pediatric patients presenting unscheduled at two Cambodian hospitals, July-August 2012

\begin{tabular}{|c|c|}
\hline Characteristic & $\begin{array}{l}\text { No. (\%) of } \\
\text { patients }^{\mathrm{a}, \mathrm{b}}\end{array}$ \\
\hline All patients & 867 \\
\hline \multicolumn{2}{|l|}{ Demographic characteristic } \\
\hline \multicolumn{2}{|l|}{ Site of presentation } \\
\hline Battambang Provincial Hospital & $550(63.4)$ \\
\hline Sampov Meas Provincial Hospital & $317(36.6)$ \\
\hline \multicolumn{2}{|l|}{ Age } \\
\hline Age in years, mean (SD) & $5.65(4.8)$ \\
\hline Infant (<1 year) & $136(15.7)$ \\
\hline Young child ( $1-5$ years) & $374(43.1)$ \\
\hline Child (6-10 years) & $182(21.0)$ \\
\hline Older child (11-13 years) & $98(11.3)$ \\
\hline Teen (14-17 years) & $77(8.9)$ \\
\hline \multicolumn{2}{|l|}{ Gender } \\
\hline Female & $396(45.7)$ \\
\hline Male & $471(54.3)$ \\
\hline \multicolumn{2}{|l|}{ Socioeconomic characteristic } \\
\hline Patient had low-income health insurance ${ }^{c}$ & $444(51.2)$ \\
\hline \multicolumn{2}{|l|}{ Travel to hospital } \\
\hline \multicolumn{2}{|l|}{ Time } \\
\hline Time in hours, median (IQR) & $0.5(0.3-1.0)$ \\
\hline Time $<0.5 \mathrm{~h}$ & $482(55.6)$ \\
\hline Time $0.5-2 \mathrm{~h}$ & $326(37.6)$ \\
\hline Time $>2 \mathrm{~h}$ & $52(6.0)$ \\
\hline \multicolumn{2}{|l|}{ Distance } \\
\hline Distance in kilometers, median (IQR) & $10(3.0-30.0$ \\
\hline \multicolumn{2}{|l|}{ Presentation } \\
\hline \multicolumn{2}{|l|}{ Time of arrival } \\
\hline Daytime Monday to Friday (i.e., 07.00-17.00) & $765(88.2)$ \\
\hline Overnight Sunday to Friday (i.e., $17.00-07.00$ ) & $68(7.8)$ \\
\hline Weekend (i.e., Friday 17.00 to Sunday 17.00 ) & $34(3.9)$ \\
\hline \multicolumn{2}{|l|}{ Care before presentation } \\
\hline Transferred from another healthcare facility & $163(18.8)$ \\
\hline Referred by an external medical provider & $81(9.3)$ \\
\hline \multicolumn{2}{|l|}{ Prior care of those not transferred or referred } \\
\hline Prior care received in the previous $48 \mathrm{~h}$ & $79(9.1)$ \\
\hline Prior care received more than $48 \mathrm{~h}$ earlier & $65(7.5)$ \\
\hline No prior care & $468(54.0)$ \\
\hline \multicolumn{2}{|l|}{ Symptom duration } \\
\hline Sudden $(<24$ h) & $266(30.7)$ \\
\hline Recent ( $1-3$ days) & $340(39.2)$ \\
\hline Sub-acute (4-14 days) & $206(23.8)$ \\
\hline Chronic (> 14 days) & $49(5.7)$ \\
\hline
\end{tabular}

Table 1 Pediatric patients presenting unscheduled at two Cambodian hospitals, July-August 2012 (Continued)

\begin{tabular}{|c|c|}
\hline Characteristic & $\begin{array}{l}\text { No. (\%) of } \\
\text { patients }{ }^{\mathrm{a}, \mathrm{b}}\end{array}$ \\
\hline \multicolumn{2}{|l|}{ Time before seeking any medical care } \\
\hline Median time before seeking care in days (IQR) & $1.5(0.6-3)$ \\
\hline$<=24 \mathrm{~h}$ & $339(39.1)$ \\
\hline$>24 \mathrm{~h}$ & $505(58.3)$ \\
\hline \multicolumn{2}{|l|}{ Examination findings } \\
\hline \multicolumn{2}{|l|}{ Respiratory rate, blood pressure, and heart rate ${ }^{d}$} \\
\hline $\begin{array}{l}\text { Abnormal respiratory rate, blood pressure, } \\
\text { or heart rate }\end{array}$ & $405(46.7)$ \\
\hline Abnormal respiratory rate & $224(25.8)$ \\
\hline Abnormal blood pressure & $153(17.6)$ \\
\hline Abnormal heart rate & $217(25.0)$ \\
\hline $\begin{array}{l}\text { Respiratory rate, blood pressure, } \\
\text { or heart rate not recorded }\end{array}$ & $216(24.9)$ \\
\hline \multicolumn{2}{|l|}{ Temperature } \\
\hline Low temperature $\left(<36^{\circ} \mathrm{C}\right)$ & $80(9.2)$ \\
\hline High temperature $\left(>38^{\circ} \mathrm{C}\right)$ & $153(17.7)$ \\
\hline Temperature not recorded & $213(24.6)$ \\
\hline \multicolumn{2}{|l|}{ Pain } \\
\hline Pain documented as present & $254(29.3)$ \\
\hline
\end{tabular}

$I Q R$ interquartile range, $S D$ standard deviation

${ }^{a}$ Number of patients and percentages, unless otherwise stated

${ }^{b}$ Due to missing values for individual patients, not all categories sum to $100 \%$

${ }^{\mathrm{c}}$ Except for more affluent patients who had private health insurance, most patients paid in cash

${ }^{\mathrm{d}}$ See Additional file 2: Table S2 for age-appropriate vital signs used

another health provider $(\mathrm{OR}=7.8, \mathrm{CI} 4.7-13.0)$, lowincome health insurance (proxy for low socioeconomic status) $(\mathrm{OR}=1.5$, CI 1.1-2.1), seeking care prior to $48 \mathrm{~h}$ before presentation without transfer/referral $(\mathrm{OR}=2.4$, 95\% CI 1.-4.5), seeking care within $48 \mathrm{~h}$ of presentation without transfer/referral ( $\mathrm{OR}=2.5$, CI 1.4-4.5), symptom onset within $24 \mathrm{~h}$ of seeking care (2.1, CI 1.5-3.1), abnormal temperature ( $\mathrm{OR}=1.5, \mathrm{CI} 1.0-2.2)$, abnormal heart rate, respiratory rate, and/or blood pressure $(\mathrm{OR}=3.2$, CI 2.3-4.6), and chief complaint of abdominal pain $(\mathrm{OR}=2.9 ; 95 \%$ CI $1.7-5.0)$ or fever $(\mathrm{OR}=1.4$, CI 1.0-2.0); a decreased risk of admission was associated with skin-related chief complaints $(\mathrm{OR}=0.4$; CI $0.2-0.5)$, such as rashes or blisters, and respiratory problems $(\mathrm{OR}=0.4$; CI $0.3-0.6)$, such as cough $(n=793$; $c$-statistic $=0.81)$.

\section{Dermatologic and fever complaints sub-analysis}

A country-wide outbreak of enterovirus strain EV-71 occurred during the study. Qualitatively, many more children than expected presented with fever and/or skin complaints, such as rash or blisters, which are common 


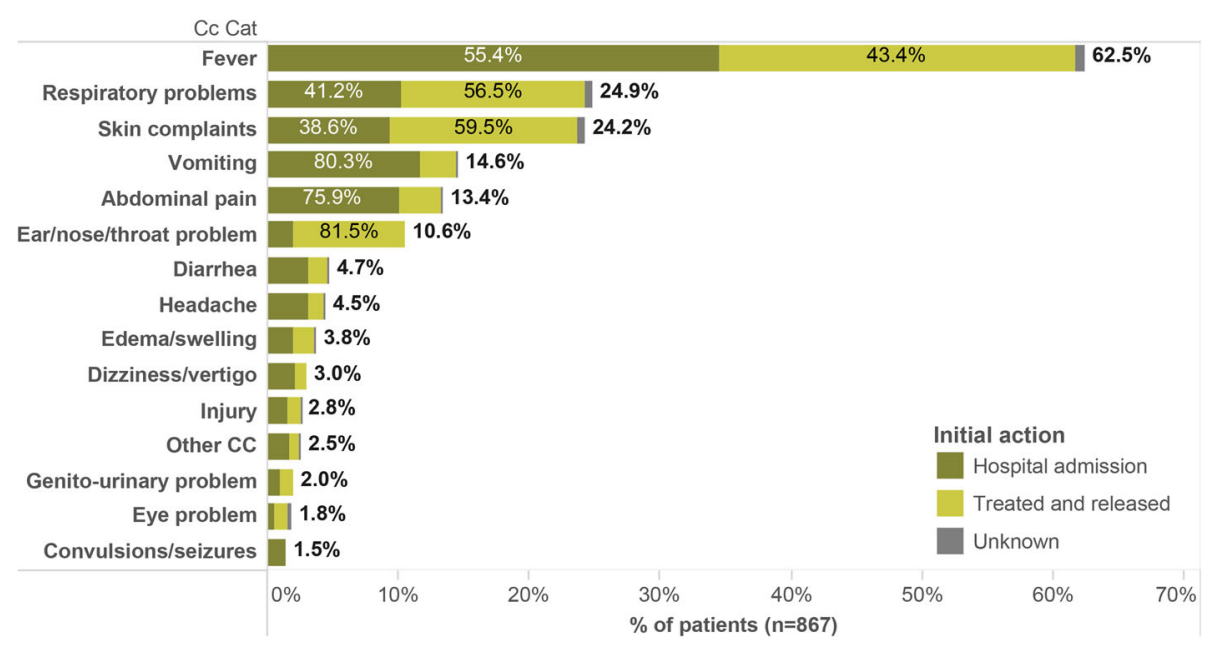

Fig. 1 Chief complaints and initial actions for children presenting without appointment at two Cambodian hospitals. Only the top 15 chief complaints are displayed. A maximum of three chief complaints per patient were recorded. Includes data for July-August 2012

symptoms of EV-71. The number of patients presenting with skin complaints and fever increased sharply during the study period before gradually decreasing, coinciding with timing of the EV-71 outbreak (Fig. 2).

\section{Discussion}

This study describes the characteristics, chief complaints, management, and health outcomes of children seeking unscheduled acute care at two public referral hospitals in Cambodia. Our findings provide essential information to inform emergency care provider education and guide development of emergency care systems in Cambodia and other LMICs.

Top chief complaints in this study (fever, respiratory problems, abdominal pain, vomiting, and diarrhea) are similar to other published reports characterizing unscheduled or ED pediatric presentations in other LMICs $[6,8,19,20]$. The top five chief complaints in our population accounted for $75 \%$ of all reported complaints. In contrast to several other studies, the percentage of ED visits attributed to trauma in this study (2.8\%) was substantially lower. For example, trauma among children presenting for emergency care accounted for $27-29 \%$ of visits in Korea and Malaysia and 25\% of visits in the USA $[8,21,22]$. The underrepresentation of trauma in our study may have been due to the presence of an NGO-run trauma hospital near the study sites, which might have received the bulk of trauma patients. The top chief complaints in our and other studies often have infectious etiologies [23, 24]. While infections such as pneumonia, diarrhea, and malaria are the leading contributors to mortality in children under 5 years worldwide, [25] infectious symptoms also account for a large percentage of ambulatory care-sensitive conditions [26].
Of the top ten chief complaints identified in the present study, only half match the list of top complaints documented in an analogous population of adults [5]. These results mirror data from EDs in the USA, which similarly show that the frequency and distribution of pediatric chief complaints differ markedly from adults [9]. The unique distribution and frequency of presentations in children with undifferentiated conditions compared to adults in LMICs underscores the need for specialty training that specifically addresses pediatric emergencies.

Regarding pre-hospital care, only $2.9 \%$ of patients in our study arrived by ambulance, compared to $15-17 \%$ in other studies in LMICs [27, 28]. Low frequency of ambulance transport-despite more than one quarter of all unscheduled patients coming from another care provider-reflects the relative underdevelopment of Cambodia's regional pre-hospital and EMS systems. Currently, laypeople provide most patient transfers in Cambodia. Low-cost, community-based interventions aimed at equipping laypeople with first aid skills and facilitating transport have shown promise in South Africa [29].

Vital signs, which provide low-cost, objective data that can help prioritize patients during triage and guide initial therapy, were not measured or recorded in nearly one quarter of enrolled patients. Emphasis on obtaining vital signs should be included in all training for emergency care providers to improve management for pediatric patients presenting for acute care.

We also observed that respiratory complaints and fever were the two most common complaints, yet diagnostic chest X-rays (CXR) were infrequently performed, even for admitted patients. Furthermore, despite the high prevalence of tuberculosis in Cambodia $(817 / 100,000$ population) relative to other countries in 
Table 2 Diagnostics and interventions within $48 \mathrm{~h}$ in children presenting unscheduled at two Cambodian hospitals

\begin{tabular}{|c|c|c|}
\hline $\begin{array}{l}\text { Diagnostic test } \\
\text { or intervention }\end{array}$ & $\begin{array}{l}\text { No. }(\%) \text { presenting, } \\
(n=867)\end{array}$ & $\begin{array}{l}\text { No (\%) admitted }{ }^{b, c} \\
(n=445)\end{array}$ \\
\hline \multicolumn{3}{|l|}{ Diagnostic test } \\
\hline Any test & $278(32.1)$ & $263(59.1)$ \\
\hline Laboratory test & $270(31.1)$ & $259(58.2)$ \\
\hline Diagnostic imaging & $19(2.2)$ & $14(3.2)$ \\
\hline $\begin{array}{l}\text { Diagnostic peritoneal } \\
\text { lavage }\end{array}$ & $2(0.2)$ & $2(0.5)$ \\
\hline Ultrasound scanning & $1(0.1)$ & $1(0.2)$ \\
\hline \multicolumn{3}{|l|}{ Medication administered } \\
\hline Any medication & 707 (81.6) & $343(77.1)$ \\
\hline $\begin{array}{l}\text { Analgesic } \\
\text { (excluding aspirin) }\end{array}$ & $602(69.4)$ & $288(64.7)$ \\
\hline Antibiotic & $412(47.5)$ & $172(38.7)$ \\
\hline Antiparasitic & $62(7.2)$ & $7(1.6)$ \\
\hline $\begin{array}{l}\text { Drug administered } \\
\text { by nebulizer }\end{array}$ & $29(3.3)$ & $18(4.0)$ \\
\hline Antimalarial & $7(0.8)$ & $7(1.6)$ \\
\hline Aspirin & $3(0.4)$ & 0 \\
\hline Antituberculosis drug & $1(0.1)$ & 0 \\
\hline Other & 255 (29.4) & 94 (21.1) \\
\hline
\end{tabular}

Other interventions

$\begin{array}{lll}\begin{array}{l}\text { Any intervention other } \\ \text { than medication }\end{array} & 380(43.8) & 356(80.0) \\ \text { Intravenous fluids } & 353(40.7) & 345(77.5) \\ \text { Intravenous medication } & 42(4.8) & 41(9.2) \\ \text { Emergency cooling } & 35(4.0) & 35(7.9) \\ \text { Oxygen therapy } & 33(3.8) & 29(6.5) \\ \text { Oral hydration } & 29(3.3) & 19(4.3) \\ \text { Wound closure } & 13(1.5) & 11(2.5) \\ \text { Urethral catheterization } & 6(0.7) & 6(1.3) \\ \text { Gastric decontamination } & 6(0.7) & 6(1.3) \\ \text { Dental procedures } & 4(0.5) & 0 \\ \text { Blood transfusion } & 3(0.3) & 2(0.4) \\ \text { Endotrachial intubation } & 3(0.3) & 3(0.7)\end{array}$

${ }^{a}$ Categories may sum to more than $100 \%$ because some patients had more than one diagnostic test or intervention

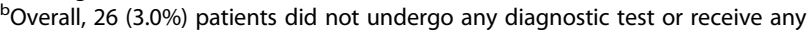
intervention and, for $82(9.5 \%)$ of patients, no information on diagnostic tests or interventions was collected

'Overall, four (0.9\%) admitted patients did not undergo any diagnostic test or receive any intervention and, for $49(11.0 \%)$ patients, no information on diagnostic tests or interventions was collected

the South-East Asia Region [30], only one patient received treatment for tuberculosis. These findings indicate that further studies are needed to assess the diagnostic evaluation among children with respiratory complaints to ensure that tuberculosis and other respiratory illnesses are recognized and adequately treated.
Table 3 Outcomes and follow-up in children presenting unscheduled at two Cambodian hospitals, July-August 2012

\begin{tabular}{|c|c|}
\hline Outcome & $\begin{array}{l}\text { No. (\%) presenting } \\
(n=867)^{\mathrm{a}}\end{array}$ \\
\hline \multicolumn{2}{|l|}{ Initial visit } \\
\hline Patient treated and released & $403(46.5)$ \\
\hline Patient transferred to another facility ${ }^{\mathrm{b}}$ & $8(0.9)$ \\
\hline $\begin{array}{l}\text { Patient left hospital without being seen } \\
\text { or against medical advice }\end{array}$ & $4(0.5)$ \\
\hline Patient died within $24 \mathrm{~h}$ of presentation & $2(0.2)$ \\
\hline Patient admitted & $445(51.3)$ \\
\hline $\begin{array}{l}\text { Length of stay of patients admitted in days, } \\
\text { median (IQR) }\end{array}$ & $3(2-4)$ \\
\hline \multicolumn{2}{|l|}{ 48-h follow-up } \\
\hline Patient followed up at $48 \mathrm{~h}$ & $719(82.9)$ \\
\hline Patient remained functionally impaired ${ }^{c, d}$ & $362(50.3)$ \\
\hline $\begin{array}{l}\text { Patient seen by another healthcare } \\
\text { provider after discharge }{ }^{c}\end{array}$ & $45(5.6)$ \\
\hline Patient died between 24 and $48 \mathrm{~h}^{\mathrm{c}}$ & $4(0.6)$ \\
\hline \multicolumn{2}{|l|}{ 14-day follow-up } \\
\hline Patient followed up at 14 days & $649(74.9)$ \\
\hline Patient remained functionally impaired ${ }^{d,}$ e & $44(6.8)$ \\
\hline $\begin{array}{l}\text { Patient seen by another healthcare } \\
\text { provider after discharge }\end{array}$ & $86(13.3)$ \\
\hline Patient died between $48 \mathrm{~h}$ and 14 days $\mathrm{e}^{\mathrm{e}}$ & $1(0.2)$ \\
\hline \multicolumn{2}{|l|}{ Cumulative outcomes } \\
\hline Patient had any surgical procedure $\mathrm{e}^{\mathrm{e}}$ & $22(3.4)$ \\
\hline Cumulative mortality at 14 days ${ }^{\mathrm{e}}$ & $7(1.1)$ \\
\hline
\end{tabular}

$I Q R$ interquartile range

${ }^{a}$ Number presenting and percentage, unless otherwise stated

${ }^{b}$ Includes only patients transferred to another facility without first being admitted and receiving care

'Percentage of the 719 patients followed up for 48 hours

${ }^{\mathrm{d}}$ Functional impairment was defined as significant pain, significant limitation in performing daily activities, confinement to bed, or a comatose state

'Percentage of the 649 patients followed up for 14 days

Finally, we observed a high admission rate (51.3\%) compared to other published pediatric studies (15-35\%) $[8,19,20]$. Since our 48 -h mortality rate, a marker of patient severity, of $0.7 \%$, was comparable to the $24-\mathrm{h}$ mortality rate reported in other studies examining similar patient populations, it is likely that a portion of the admitted patients did not require inpatient care $[20,27]$. Inappropriate admissions unnecessarily consume healthcare resources and place patients at increased risk for hospital-acquired infections, a known hazard in LMICs [31]. Such a high admission rate coupled with a relatively low mortality rate reinforces the need for a more organized emergency and acute care system in which providers are equipped to rapidly initiate diagnostic workup, provide timely treatment, and make informed decisions about patient disposition. 


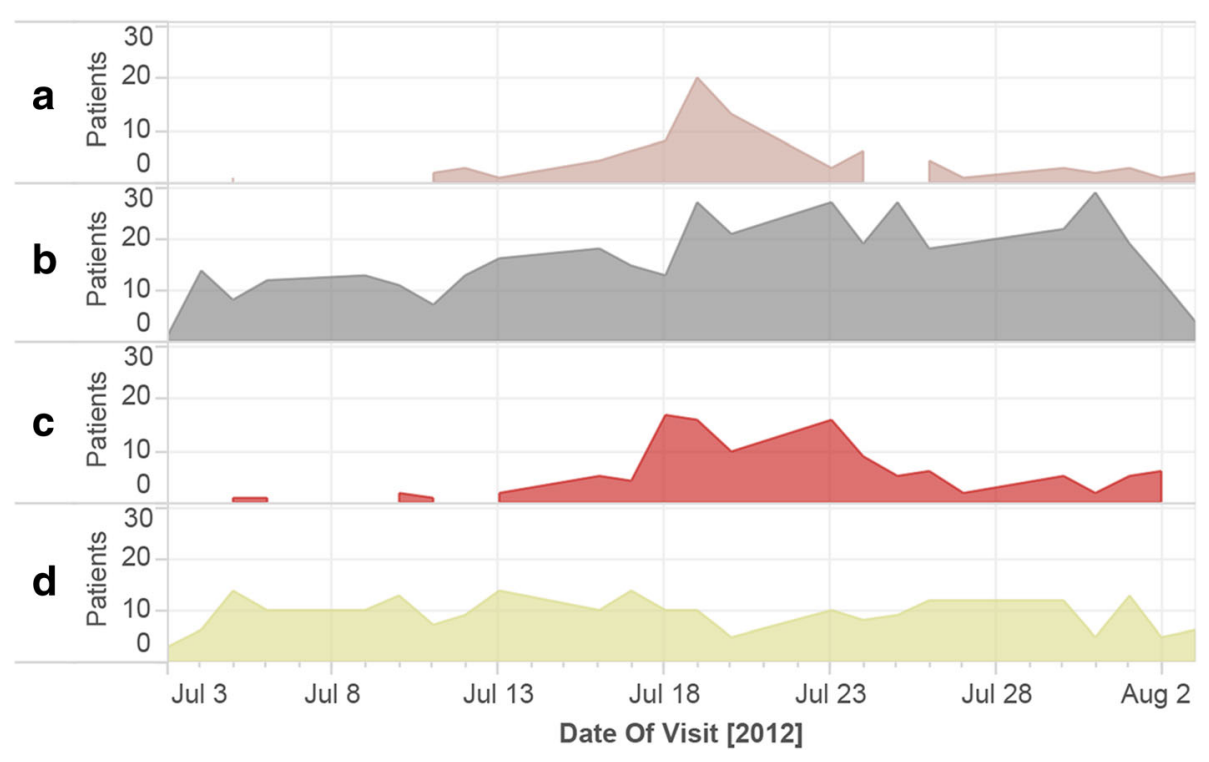

Fig. 2 Presentation of children with specific chief complaints. a Skin-related chief complaints without fever complaint. b Chief complaint of fever without skin-related complaint. c Both skin-related and fever chief complaint. d All other chief complaints other than skin-related or fever. Patients who presented during weekends are not included, due to low weekend enrollment

Our study found a high frequency of skin complaints and fever among enrolled patients relative to other studies. This was likely associated with the concurrent, widely publicized outbreak of a severe strain of enterovirus, EV-71. Symptoms included fever, rash or blisters inside the mouth and on the hands and feet, and, in severe cases, encephalitis and respiratory distress [32]. In mid-July 2012, the Cambodian Ministry of Health organized public education campaigns on symptoms of EV-71, recommending medical care for children with severe symptoms [33, 34]. The increase in patient visits during the outbreak, particularly during the peak stretch from 14 July 2012 to 25 July 2012, suggests that these campaigns effectively increased health-seeking behavior among patients with relevant symptoms.

\section{Limitations}

Key limitations of this study included inability to capture seasonal variations in illness patterns during the 4-week study period or regional variation due to the close proximity of study hospitals to one another, the likely impact of the concurrent outbreak of EV-71, and lack of data collected on weekends and overnight. As stated above, the low frequency of trauma complaints at our study sites may be due to a nearby NGO-run hospital, and, therefore, is not generalizable across Cambodia.

\section{Conclusions}

As far as we know, this is the first paper to report on the epidemiology of pediatric patients presenting unscheduled to hospitals in Cambodia. This paper builds on previous work characterizing the unscheduled adult population and finds important differences. Since pediatric patients have a different spectrum and frequency of chief complaints from adults in emergency care settings, this paper finds a need for both dedicated research into the unique presentations of children and specific training for medical professionals for emergency pediatric care. For next steps, results presented here can help inform the development of chief complaint-oriented training modules for medical providers who will staff newly founded emergency departments in Cambodia and similar settings. Results may also be useful to Cambodian hospital administrators and public health officials to further inform resource allocation for patient care, for example, by ensuring hospitals have sufficient equipment for measuring vital signs or investing in the implementation of a triage system. In addition, some specific results from this study may also serve as baseline metrics for patient outcomes-including admission rate, morbidity, and mortality-against which progress can be measured as emergency medicine matures as a distinct specialty and practice in Cambodia and other LMICs.

\section{Additional files}

Additional file 1: Figure S1. Chief complaints. All chief complaints reported by patients were coded by researchers as one of the complaints listed. (PDF $1287 \mathrm{~kb}$ )

Additional file 2: Table S1. Criteria for abnormal vital signs. (PDF $171 \mathrm{~kb}$ ) 


\section{Abbreviations}

BP: Blood pressure; BPH: Battambang Provincial Hospital; DALYs: Disability-adjusted life years; ED: Emergency department; HR: Heart rate; LMICs: Low- and middle-income countries; REDCap: Research Electronic Data Capture; RR: Respiratory rate; SMPV: Sampov Meas Provincial Hospital; WHO: World Health Organization

\section{Acknowledgements}

We thank Phan Chamroen, Van Chamroeun, Chan Dara, Sam Ol Vichet, Liong Sao, Soy Serey, Chuum Sophea, and Ou Souvichet, who provided language interpretation and assisted with the data collection. We also thank Anne Tecklenburg Strehlow for her comments on the manuscript and Andrew Martin for his technical support. We are grateful to the staff of the Battambang and Sampov Meas provincial hospitals who provided guidance on study logistics. Last but not least, we acknowledge with gratitude all of the patients and families who participated.

\section{Funding}

We obtained funding through the Stanford MedScholars Program and United States Agency for International Development under the Better Health Services project (Cooperative agreement no. 442-A-00-09-00007-00; http://www.urc-chs.com/projects/better-health-services-bhs).

\section{Availability of data and materials}

Original data are stored in Dryad (DOI https://doi.org/10.5061/dryad.7v8c4) and will be made publicly available upon publication.

\section{Authors' contributions}

MAY contributed to the protocol development, patient enrollment and interviews, and data analysis and had primary responsibility for writing the manuscript. MCS shared primary responsibility for the protocol development and also contributed to the outcome assessment and writing the manuscript. LDY contributed to the protocol development, patient enrollment and interviews, and writing the manuscript. EAP contributed to the protocol development, data analysis, and writing the manuscript. JLW contributed to the protocol development and writing the manuscript. KS contributed to the protocol development. YS contributed to the protocol development. LA contributed to the protocol development and patient enrollment and interviews. RB contributed to the protocol development and patient enrollment and interviews. CG contributed to the protocol development. SVM shared primary responsibility for the protocol development and also contributed to the outcome assessment and writing the manuscript. All authors read and approved the final manuscript.

\section{Ethics approval and consent to participate}

The Institutional Review Boards at Stanford University School of Medicine (IRB-24735) and the Cambodian Ministry of Health approved this study.

\section{Competing interests}

The authors declare that they have no competing interests.

\section{Publisher's Note}

Springer Nature remains neutral with regard to jurisdictional claims in published maps and institutional affiliations.

\footnotetext{
Author details

${ }^{1}$ Department of Emergency Medicine, UCSF Fresno Center for Medical Education and Research, 155 N Fresno St, Fresno, CA 93701, USA. ${ }^{2}$ Department of Emergency Medicine, Stanford University School of Medicine, 300 Pasteur Drive, Stanford, CA 94305, USA. 'Department of Internal Medicine, Boston University Medical Center, Boston, MA, USA ${ }^{4}$ USAID Central Asia, Almaty, Kazakhstan. ${ }^{5}$ University Research Co., LLC, Centre for Human Services, Phnom Penh, Cambodia. ${ }^{6} \mathrm{GIZ}$-Social Health Protection Program Cambodia, Phnom Penh, Cambodia. ${ }^{7}$ University of Arizona College of Medicine-Phoenix, Phoenix, AZ, USA. ${ }^{8}$ UCSF Benioff Children's Hospital Oakland, Oakland, CA, USA.
}

Received: 1 September 2017 Accepted: 15 February 2018

Published online: 13 March 2018

\section{References}

1. Kobusingye OC, Hyder AA, Bishai D, et al. Chapter 68: emergency medical services. In: Jamison DT, Breman JG, Measham AR, et al, editors. Disease control priorities in developing countries. Second ed. New York, NY: Oxford University Press; 2006. p.1261-1280.

2. Heuveline P. 'Between one and three million': towards the demographic reconstruction of a decade of Cambodian history (1970-79). Popul Stud (Camb). 1998:52:49-65.

3. Heng MB, Key PJ. Cambodian health in transition. BMJ. 1995;311:435-7.

4. Mowafi $\mathrm{H}$, Dworkis $\mathrm{D}$, Bisanzo $\mathrm{M}$, et al. Making recording and analysis of chief complaint a priority for global emergency care research in low-income countries. Acad Emerg Med. 2013;20:1241-5.

5. Yan LD, Mahadevan SV, Yore M, et al. An observational study of adults seeking emergency care in Cambodia. Bull World Health Organ. 2015:93:84-92

6. Goh AY, Chan TL, Abdel-Latiff ME. Paediatric utilization of a general emergency department in a developing country. Acta Paediatr. 2003;92:965-9.

7. Nawar EW, Niska RW, National Hospital XJ. Ambulatory medical care survey: 2005 emergency department summary. Advance data from vital and health statistics; no. 386. Hyattsville, MD: National Center for Health Statistics; 2007.

8. Kwak YH, Kim do K, Jang HY. Utilization of emergency department by children in Korea. J Korean Med Sci. 2012;27:1222-8.

9. National hospital ambulatory medical care survey: 2011 emergency department summary tables. Centers for Disease Control and Prevention (CDC). 2011. http://www.cdc.gov/nchs/data/ahcd/nhamcs_emergency/ 2011_ed_web_tables.pdf. Accessed 4 Aug 2015.

10. Management of the child with a serious infection or severe malnutrition: guidelines for care at the first-referral level in developing countries. Geneva: World Health Organization. 2000. http://apps.who.int/iris/bitstream/10665/ 42335/1/WHO_FCH_CAH_00.1.pdf. Accessed from 4 Mar 2016.

11. Gove S, Tamburlini G, Molyneux E, et al. Development and technical basis of simplified guidelines for emergency triage assessment and treatment in developing countries. WHO integrated Management of Childhood IIIness (IMCI) Referral Care Project. Arch Dis Child 1999; 81:473-477.

12. Baker T. Pediatric emergency and critical care in low-income countries. Paediatr Anaesth. 2009;19:23-7.

13. English M, Esamai F, Wasunna A, et al. Delivery of paediatric care at the first-referral level in Kenya. Lancet. 2004;364:1622-9.

14. Reyburn H, Mwakasungula E, Chonya S, et al. Clinical assessment and treatment in paediatric wards in the north-east of the United Republic of Tanzania. Bull World Health Organ. 2008:86:132-9.

15. Nolan T, Angos P, Cunha AJ, et al. Quality of hospital care for seriously ill children in less-developed countries. Lancet. 2001;357:106-10.

16. Health Service Delivery Profile-Cambodia 2012. World Health Organization and Ministry of Health, Cambodia. 2012. http://www.wpro.who.int/health_ services/service delivery_profile_cambodia.pdf. Accessed 4 Mar 2016.

17. Annual Health Statistics Report 2012. Phnom Penh: Department of Planning and Health Information, Ministry of Health Cambodia. 2012 http://www.hiscambodia.org/public/fileupload/Annual_Statistic_2012.pdf. Accessed 4 Mar 2016

18. Harris PA, Taylor $\mathrm{R}$, Thielke $\mathrm{R}$, et al. Research electronic data capture (REDCap) - a metadata-driven methodology and workflow process for providing translational research informatics support. J Biomed Inform. 2009:42:377-81.

19. Bazaraa HM, El Houchi S, Rady HI. Profile of patients visiting the pediatric emergency service in an Egyptian university hospital. Pediatr Emerg Care. 2012;28:148-52.

20. Salaria M, Singhi SC. Profile of patients attending pediatric emergency service at Chandigarh. Indian J Pediatr. 2003;70:621-4.

21. Goh AY, Abdel-Latif M, Lum LC, Abu-Bakar MN. Outcome of children with different accessibility to tertiary pediatric intensive care in a developing country - a prospective cohort study. Intensive Care Med. 2003:29:97-102.

22. Rasooly IR, Mullins PM, Alpern ER, et al. US emergency department use by children, 2001-2010. Pediatr Emerg Care. 2014:30:602-7.

23. Oguonu T, Adaeze Ayuk $C$, et al. Pattern of respiratory diseases in children presenting to the paediatric emergency unit of the University of Nigeria Teaching Hospital, Enugu: a case series report. BMC Pulm Med. 2014;14:101. 
24. Marin JR, Alpern ER. Abdominal pain in children. Emerg Med Clin North Am. 2011;29:401-28

25. Liu L, Oza S, Hogan D, et al. Global, regional, and national causes of child mortality in 2000-13, with projections to inform post-2015 priorities: an updated systematic analysis. Lancet. 2015;385:430-40.

26. Jaeger MW, Ambadwar PB, King AJ, et al. Emergency care of children with ambulatory care sensitive conditions in the United States. J Emerg Med. 2015:49:729-39.

27. Kiser MM, Samuel JC, Mclean SE, et al. Epidemiology of pediatric injury in Malawi: burden of disease and implications for prevention. Int J Surg. 2012:10:611-7.

28. Hyder AA, Sugerman DE, Puvanachandra P, et al. Global childhood unintentional injury surveillance in four cities in developing countries: a pilot study. Bull World Health Organ. 2009;87:345-52.

29. Sun $J H$, Shing $R$, Twomey $M$, et al. A strategy to implement and support pre-hospital emergency medical systems in developing, resource-constrained areas of South Africa. Injury. 2014;45:31-8.

30. World Health Statistics, 2013. World Health Organization. 2013. http://www.who.int/gho/publications/world_health_statistics/EN_ WHS2013_Full.pdf. Accessed 4 Mar 2016.

31. Allegranzi B, Nejad SB, Combescure C, et al. Burden of endemic health-care-associated infection in developing countries: systematic review and meta-analysis. Lancet. 2011;377:228.

32. World Health Organization-Western Pacific Regional Office. Phnom Penh: Severe hand, foot and mouth disease killed Cambodian children. 2012. http://www.wpro.who.int/mediacentre/news/2012/20120727/en/. Accessed 12 Aug 2015.

33. Biswas T. Enterovirus 71 causes hand, foot and mouth disease outbreak in Cambodia. Natl Med J India. 2012;25:316.

34. Seiff A. Cambodia unravels cause of mystery illness. Lancet. 2012;380:206

\section{Submit your manuscript to a SpringerOpen ${ }^{\circ}$ journal and benefit from:}

- Convenient online submission

- Rigorous peer review

- Open access: articles freely available online

- High visibility within the field

- Retaining the copyright to your article

Submit your next manuscript at $>$ springeropen.com 